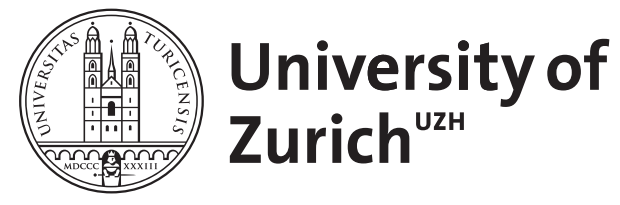
Archive

University of Zurich

University Library

Strickhofstrasse 39

CH-8057 Zurich

www.zora.uzh.ch

Year: 2009

Traumafokussierte kognitive Verhaltenstherapie mit einem Kriegsopfer: Ein Fallbericht

Müller, J

DOI: https://doi.org/10.1159/000228932

Posted at the Zurich Open Repository and Archive, University of Zurich ZORA URL: https://doi.org/10.5167/uzh-20622

Journal Article

Published Version

Originally published at:

Müller, J (2009). Traumafokussierte kognitive Verhaltenstherapie mit einem Kriegsopfer: Ein Fallbericht. Verhaltenstherapie, 19(3):177-183.

DOI: https://doi.org/10.1159/000228932 


\title{
Traumafokussierte kognitive Verhaltenstherapie mit einem Kriegsopfer: Ein Fallbericht
}

\author{
Julia Müller \\ Ambulatorium für Folter- und Kriegsopfer, Psychiatrische Poliklinik, Universitätsspital Zürich, Schweiz
}

\section{Schlüsselwörter \\ Posttraumatische Belastungsstörung (PTSD) · Krieg · \\ Asylbewerber · Flüchtling · Kognitive \\ Verhaltenstherapie (KVT) · Exposition · Stabilisierung}

\section{Zusammenfassung}

Zur Behandlung posttraumatischer Belastungsstörungen (PTSD) gelten traumafokussierte Verfahren international als Methode der Wahl. Erste Studien legen nahe, dass diese Methoden auch bei traumatisierten Asylsuchenden anwendbar und effizient sind. Zusätzlich zu ihren meist schwer ausgeprägten Symptomen leben Asylsuchende in anhaltend unsicheren Lebenssituationen. Letztere können im Therapieverlauf wiederholte Krisen auslösen, die eine Unterbrechung der Traumakonfrontation nötig machen. Dieser Fallbericht beschreibt den Verlauf und die Ergebnisse einer traumafokussierten kognitiven Verhaltenstherapie (KVT) mit einem 30-jährigen Bosnier, der in der Schweiz Asyl beantragt hat. Die 69 Sitzungen dauernde KVT enthielt die Elemente Therapievorbereitung, Alltagsbewältigung, Expositionen in sensu und in vivo sowie Ressourcenaktivierung. Die deutsche Version der PTSD Symptom Scale wurde vor und nach der Haupttherapiephase sowie nach der Therapieabschlussphase abgenommen. Die zu Therapiebeginn stark ausgeprägte PTSD-Symptomatik nahm im Verlauf der Sitzungen deutlich ab. Nach der Expositionsphase verbesserten sich die Symptome weiterhin. In der 18-Monatskatamnese war der Patient störungsfrei. Der Fallbericht zeigt beispielhaft, dass evidenzbasierte traumafokussierte KVT inklusive früher Traumaexposition zur Behandlung traumatisierter Asylsuchender anwendbar ist und die Belastung deutlich reduzieren kann - auch wenn zum Behandlungszeitpunkt erhebliche Postmigrationsstressoren bestehen. Gleichzeitig illustriert der Bericht, dass die Traumakonfrontationen möglicherweise wiederholt durch Phasen stabilisierender Interventionen unterbrochen werden müssen.

\author{
Key Words \\ Posttraumatic stress disorder (PTSD) - War - Asylum \\ seeker - Refugee - Cognitive behavioural therapy (CBT) . \\ Exposure $\cdot$ Stabilisation
}

\section{Summary}

Trauma-Focused Cognitive Behavioural Therapy with a Victim of War: A Case Report

Internationally, trauma-focused interventions are acknowledged as the method of choice in the treatment of posttraumatic stress disorder (PTSD). Initial evidence on the use of these methods in traumatised asylum seekers does exist. In addition to their often severe symptoms, these patients do experience continuously insecure living conditions. These additional problems may provoke sudden crises that make it necessary to interrupt traumafocused interventions. The present case report describes the procedure and the results of trauma-focused cognitive behavioural therapy (CBT) in a 30-year-old male Bosnian seeking asylum in Switzerland. The patient received 69 sessions of CBT involving preparation for the intervention, mastery of daily problems, imaginal exposure and exposure in vivo, and reactivation of resources. The German PTSD Symptom Scale was completed at pretreatment, after the main therapy phase, and after the termination phase of the therapy. While the patient was highly symptomatic of PTSD at the beginning of treatment, symptoms decreased during therapy. The symptoms declined further after treatment, and 18 months after termination of the exposure phase, he no longer met criteria for PTSD diagnosis. This case report demonstrates that trauma-focused CBT may be applicable and effective in the treatment of traumatised asylum seekers despite additional post-migration stressors during therapy. The case also illustrates how trauma exposure needs to be repeatedly interrupted by phases of stabilising interventions.

\section{KARGER}

Fax +497614520714

Information@Karger.de

www.karger.com (c) 2009 S. Karger GmbH, Freiburg 


\section{Einleitung}

Ende 2006 befanden sich weltweit knapp 33 Millionen Menschen in flüchtlingsähnlichen Situationen [United Nations High Commissioner for Refugees, 2008]. Flüchtlinge haben ein deutlich erhöhtes Risiko, an somatischen Krankheiten [Gavagan und Brodyaga, 1998] und psychischen Störungen [Fazel et al., 2005; Hollifield et al., 2002] zu leiden; dies umso mehr, wenn traumatische Erfahrungen vorliegen. In den Hochrisikopopulationen der Flüchtlinge und Asylbewerber sind posttraumatische Belastungsstörungen (PTSD) verglichen mit den Lebenszeitprävalenzen der Normalbevölkerung westlicher Länder (1,0-1,3\%) [Davidson et al., 1991; Perkonigg et al., 2000] signifikant häufiger (17-65\%) [z.B. de Jong et al., 2001; Mollica et al., 1999; Turner et al., 2003]. Für im Exil lebende Flüchtlinge aus dem ehemaligen Jugoslawien werden PTSDRaten von 30-60\% berichtet [Ai et al., 2002; Thulesius und Hakansson, 1999; von Lersner et al., 2008; Weine et al., 1998]. Miller et al. [2005] erklären diese uneinheitlichen Prävalenzen mit ungleichen Traumadosen und verschiedenen Lebensumständen der Befragten zwischen den einzelnen Studien.

Internationale Fachgesellschaften [z.B. Australian Centre for Posttraumatic Mental Health, 2007; Foa et al., 2004; National Institute for Health and Clinical Excellence (NICE), 2005] und aktuelle Überblicksarbeiten [Bisson und Andrew, 2007; Bradley et al., 2005] kommen aufgrund der Datenlage zu dem Schluss, dass traumafokussierte Verfahren, wie kognitive Verhaltenstherapie, die Methode der Wahl zur Behandlung von PTSD sind. Andere Verfahren sind weniger wirksam oder ihre Wirksamkeit ist nicht ausreichend belegt. Kontrollierte Studien zeigen, dass traumafokussierte Verfahren auch bei kriegstraumatisierten Flüchtlingen aus verschiedenen Kulturkreisen effizient sind [Bichescu et al., 2007; Hinton et al., 2005; Neuner et al., 2004; Otto et al., 2003; Paunovic und Ost, 2001], selbst wenn sie von trainierten Laien durchgeführt werden [Neuner et al., 2008]. Eine erste Studie zeigt außerdem, dass auch traumatisierte Asylbewerber mit unsicherem Aufenthaltsstatus hochwirksam traumafokussiert behandelt werden können [Schauer et al., 2006]; insgesamt ist hier die Evidenz jedoch geringer.

In den NICE-Leitlinien [2005] ist festgehalten, dass es sinnvoll sein kann, die traumafokussierte Therapie aufzuschieben, wenn die aktuelle Lebenssituation - z.B. der unsichere Aufenthaltsstatus als Asylbewerber - den Patienten sehr belastet. Es wird pragmatisch ein phasenorientiertes Vorgehen vorgeschlagen, bei welchem der traumafokussierten Phase nötigenfalls eine Stabilisierungsphase vorgeschoben werden kann. Ziel dieser vorgeschobenen Phase ist es, primäre Bedürfnisse des Patienten, wie dringende medizinische Versorgung, oder die Wohnbedingungen zu klären sowie ausreichende Sicherheitsgefühle aufzubauen. Ob und wie lange eine Stabilisierungsphase notwendig ist, wird dabei weniger von der tatsächlichen Sicherheit als vom individuellen Belastungsgrad des Patienten bestimmt [Kapitel 10.5, NICE, 2005]. Die letzte
Phase soll die Integration in die neue Gesellschaft bzw. die Reintegration in die eigene Gesellschaft verfolgen. Die Phasen müssen nicht linear verlaufen; vielmehr wird ein häufiger Wechsel zwischen den Phasen erwartet [NICE, 2005].

Verschiedene Autoren kritisieren, dass traumatisierte Kriegs- und Folteropfer in der Praxis häufig mit einer Mischung von Methoden behandelt werden, deren Wirksamkeit nicht belegt ist [Basoglu, 2006; Neuner, 2008; Schauer et al., 2006]. Insbesondere im deutschen Sprachraum werden verschiedene Positionen über die adäquate Behandlung von PTSD-Patienten vertreten [vgl. Fischer und Becker-Fischer, 2008; Neuner, 2008]. Es wird dabei kontrovers diskutiert, ob «eine Phase der Stabilisierung und Konsolidierung jeder weiteren therapeutischen Arbeit vorangehen muss» [Fischer et al., 2003, p. 204] oder ob dieses Dogma zugunsten einer möglichst raschen Traumaexposition aufgegeben werden sollte [Neuner, 2008].

Vor dem Hintergrund dieser - teils sehr polarisiert geführten - Diskussion beschreibt der vorliegende Fallbericht den Therapieverlauf eines Patienten mit schwerer Symptomatik und ungesichertem Aufenthaltsstatus sowie weiteren existenziellen Alltagsproblemen. Dabei zeigt sich die Bedeutsamkeit beider therapeutischen Strategien, Stabilisierung und Konfrontation, die flexibel an die individuellen Bedürfnisse des Patienten und an seine aktuellen Bedingungen angepasst wurden.

\section{Fallbericht}

\section{Überweisungskontext}

Der 26-jährige bosnische Asylbewerber wird zur Abklärung und Behandlung von seinem Hausarzt in die Ambulanz für Folter- und Kriegsopfer am UniversitätsSpital Zürich überwiesen. Der Patient sei während des Bosnienkrieges schwer traumatisiert worden und seit seiner Einreise in die Schweiz aufgrund schwerer Suizidalität zweimal stationär psychiatrisch behandelt worden.

\section{Psychosoziale Situation und Symptomatik zu Therapiebeginn}

Der Patient befindet sich zu Therapiebeginn (April 2004) seit etwa 6 Monaten in der Schweiz. Er lebt im Kanton Zürich in einer Massenunterkunft für neu angekommene Asylbewerber («Durchgangszentrum»). Sein Zimmer teilt er mit 6 jungen Männern verschiedener Nationalitäten. Er spricht kein Deutsch; die Sitzungen werden mithilfe einer Übersetzerin durchgeführt. Bis auf seinen in Bosnien lebenden jüngeren Bruder seien alle Familienangehörigen tot. Während zum Bruder sehr sporadisch Telefonkontakt bestehe, habe er die Beziehung zu sämtlichen bosnischen Freunden abgebrochen. Außer zum Hausarzt und zu seinem Asylanwalt habe er auch in der Schweiz keine sozialen Kontakte.

Der Patient berichtet, meistens auf seinem Bett zu sitzen und das Zimmer im Durchgangszentrum kaum zu verlassen. Er denke ständig an den Krieg und an seine Vergewaltigung in der Kaserne. Dabei habe er die Bilder dieser Ereignisse stets vor Augen. Er leide zudem unter ausgeprägten Schlafstörungen und erwache infolge von Albträumen oft schreiend und schweißgebadet. Nach einem solchen Erwachen könne er sich zunächst lange nicht bewegen. Da er sich vor sich selber ekle und beschmutzt fühle, müsse er sich anschließend ausgiebig duschen. Er sei zudem anhaltend innerlich unruhig und nervös, habe Angst vor anderen Menschen und traue niemandem. Er fühle sich vollkommen antriebslos und habe keine Ziele für seine Zukunft. 


\section{Vorhergehende Behandlungen}

Der Patient berichtet, unmittelbar nach dem Krieg kurzfristig psychiatrisch-psychopharmakologisch behandelt worden zu sein (Substanz unbekannt); dies habe ihm jedoch kaum geholfen. Ein erneuter Behandlungsversuch in der Schweiz sei nach drei Sitzungen gescheitert, da er weder zum Psychiater noch zur Übersetzerin Vertrauen habe fassen können. Infolge zweier Suizidversuche (2003/2004) sei er zweimal stationär behandelt worden. Während der ersten stationären Aufnahme sei eine Pharmakotherapie mit Antidepressiva (Paroxetin) und Neuroleptika (Olanzapin) begonnen wurden, die ambulant vom Hausarzt fortgeführt worden sei. Trotz der beruhigenden Wirkung habe er die Medikamente wegen schwerer Nebenwirkungen im März 2004 selbständig abgesetzt. Der Patient gibt an, von keinem seiner Behandler jemals auf seine traumatischen Erfahrungen angesprochen worden zu sein.

\section{Anamnese}

Der Patient wurde 1978 als vierter von 5 Söhnen in Bosnien-Herzegowina geboren. Sein Vater sei Zimmermann, seine Mutter Hausfrau gewesen. Die Familie lebte in einem Dorf und hatte eigene Landwirtschaft. Nach Aussagen des Patienten habe er bis zum Kriegsausbruch 1992 (14. Lebensjahr) eine «völlig normale» Kindheit gehabt. Er beschreibt gute Beziehungen zu den Eltern und den Brüdern. Als «eher frecher» Junge habe er viele Freunde gehabt, mit denen er viel Fußball gespielt habe. Er sei aber auch gerne zur Schule gegangen.

Durch den Krieg (1992-1995) habe sich alles geändert. Während der Belagerung seines Heimatdorfs durch die serbische Armee seien seine 3 älteren Brüder innerhalb weniger Wochen getötet worden. Die Tötungen der beiden Älteren durch Granaten habe er selbst miterlebt. Da das Dorf von der serbischen Armee eingenommen worden sei, habe die restliche Familie (Eltern, Großvater und 1 Bruder) fliehen müssen. Sie seien nach Srebrenica geflohen. Seine Mutter und der kleine Bruder hätten von dort mit dem Konvoi der Frauen und Kinder nach Tuzla ausreisen dürfen. Ihm hingegen sei die Flucht im Konvoi von den serbischen Soldaten versagt worden, da er mit 14 Jahren als Mann gegolten habe. Er habe etwa $1 \mathrm{Jahr}$ mit Vater und Großvater in Srebrenica bleiben müssen. Diese Zeit sei sehr hart gewesen. Er hätte oft gehungert. Banden hätten das Leben sehr gefährlich gemacht; die Stimmung sei insgesamt sehr angespannt gewesen. Mangels medizinischer Hilfe sei sein Großvater in Srebrenica verstorben. Nach der Eroberung von Srebrenica durch serbische Soldaten seien er und sein Vater in den Wald geflohen. Während eines serbischen Angriffs habe er seinen Vater verloren. Auf der Suche nach ihm sei er 1 Woche lang alleine im Wald durch die serbischen Linien ins Heimatdorf zurückgewandert. Nach erfolgloser Suche sei er alleine nach Tuzla geflohen. Auf diesem Weg habe er unzählige Traumatisierungen erlebt (Hinterhalte, Granatbeschüsse, Minenfelder, Tote, Leichenschändungen). In einem Tuzlaer Flüchtlingslager habe er Mutter und Bruder wieder gefunden. Gegen Kriegsende habe sich die Lebenssituation in Tuzla verbessert. Er sei, wie alle Kinder, wieder in die Schule gegangen. Da er die Kriegserlebnisse nicht habe vergessen können, sei er in Sarajevo zu einem Psychiater gegangen. Aus finanziellen Gründen habe er diese Therapie jedoch nach drei Konsultationen abbrechen müssen. In seinem 20. Lebensjahr (1998) sei seine kriegsbedingt stark veränderte Mutter plötzlich verstorben. Der Patient und sein damals 14-jähriger Bruder seien von da an auf sich alleine gestellt gewesen; sie hätten ihren Lebensunterhalt selbst finanzieren müssen. Im Jahr 2002 sei er zum Militärdienst einberufen worden (24. Lebensjahr). Dort sei er zur Strafe für seine demokratischen Ansichten von 2 nationalistischen Vorgesetzten vergewaltigt worden. Schwer verletzt sei er desertiert und mithilfe von Freunden in die Schweiz geflüchtet (2003). In der Schweiz habe man ihm bisher kein Asyl gewährt.

\section{Psychopathologischer Befund zu Therapiebeginn}

Großer, kräftiger, leicht verwahrlost wirkender Mann. Der Patient ist im Erstkontakt verschlossen und sozial zurückgezogen. Im Gespräch behält er seine Jacke an, sitzt auf der äußersten Stuhlkante und hält seinen
Rucksack auf dem Schoß. Er vermeidet Blickkontakt. Seine Orientierung, räumlich und zeitlich und zur eigenen Person, ist intakt. Der Patient zeigt keine Gedächtnisschwäche; Auffassung und Merkfähigkeit sind unauffällig. Die Konzentration ist nach maximal 30 min beeinträchtigt. Es liegen keine Hinweise für Ich-Störungen oder wahnhaftes Erleben vor. Die Denkfunktionen, inhaltlich und formal, sind intakt und kohärent. Es bestehen zudem keine Hinweise auf Impulsivität. Der Patient ist antriebsarm, niedergeschlagen und hoffnungslos gestimmt. Sein Affekt scheint nicht modulationsfähig. Die von ihm berichtete ausgeprägte Nervosität ist im Gespräch beobachtbar; der Patient ist motorisch sehr unruhig und reibt sich ständig die Hände. Bei traumarelevanten Themen wirkt der Patient gesteigert unruhig. Er gibt an, vor anderen Menschen Angst zu haben und soziale Kontakte zu vermeiden. Er sei außerdem sehr schreckhaft. Infolge von Intrusionen und nächtlichen Albträumen ekle er sich vor sich selbst und spüre seinen Körper kaum, weswegen er mehrmals am Tag kalt duschen müsse. Da er vom bosnischen Militär wegen seiner Desertion gesucht werde, habe er Panik, abgeschoben zu werden. Suizidabsichten verneint er zu Therapiebeginn.

\section{Diagnostik und Differentialdiagnostik}

Der Patient zeigt stark ausgeprägte und sichtbare Zeichen einer PTSD nach sequenzieller Traumatisierung bei - soweit bekannt - weitgehend ungestörter Frühentwicklung in der Kindheit. Er schildert unwillkürliches Wiedererleben, Vermeidungsverhalten und emotionale Betäubung sowie verschiedene Symptome der Übererregung. Im Vordergrund scheinen zu Therapiebeginn nicht die Kriegserlebnisse zu stehen, sondern die vom Patienten als äußerst beschämend erlebte Vergewaltigung. Die vorhandenen depressiven Symptome haben aufgrund ihrer Ausprägung eigenen Störungswert. Da die Verhaltensanalyse aber zeigt, dass diese Symptome direkt mit der PTSD zusammenhängen, werden sie für die Therapieplanung als sekundär betrachtet. Hingegen werden die vom Patienten berichteten zwanghaften (Duschen) und dissoziativen (Starre nach Albträumen) Symptome im Rahmen der PTSD betrachtet und nicht gesondert diagnostiziert.

\section{Diagnosen}

- F43.1: Posttraumatische Belastungsstörung,

- F.32.1: Mittelgradige depressive Episode (im Zusammenhang mit der PTSD).

\section{Testpsychologische Befunde der PTSD}

Zur PTSD-Diagnostik und zur Beobachtung des Therapieverlaufs wurde die deutsche modifizierte Version der PTSD Symptom Scale - Interview (PSS-I) [Steil und Ehlers, 1992] mithilfe der Dolmetscherin mündlich abgenommen. Die Skala erfasst neben der Häufigkeit auch die Belastung durch alle 17 PTSD-Symptome nach dem Diagnostischen und Statistischen Manual Psychischer Störungen (DSM-IV) [American Psychiatric Association, 1996]. Die beiden je 4-stufigen, voneinander unabhängigen Skalen reichen von 0 (Symptom ist nicht aufgetreten/belastend) bis 3 (Symptom ist fast immer aufgetreten/sehr belastend). Symptome $\geq 1$ gelten als klinisch relevant [Foa et al., 1993]. Eine PTSD wird diagnostiziert, wenn mindestens 1 Wiedererlebens-, 3 Vermeidungs- und 2 Hyperarousalsymptome mindestens 1 Monat vorhanden sind [American Psychiatric Association, 1996]. Die Ergebnisse der PSS-Diagnostik zu verschiedenen Messzeitpunkten sind in Tabelle 1 dargestellt.

\section{Verhaltensanalyse}

Der Patient liegt im Durchgangszentrum auf seinem Bett. Das Geräusch von Schritten auf der Metalltreppe vor seinem Zimmer $\left(S_{\text {extern }}\right)$ triggert Erinnerungen an die in der Kaserne erlebte Vergewaltigung $\left(\mathrm{R}_{\mathrm{kog}}\right)$. Die damals empfundene Hilflosigkeit und die resultierenden Grundannahmen «Die Welt ist gefährlich»/«Ich bin kaputt» werden reaktiviert $(\mathrm{O})$. Intrusionen und Flashbacks von der Vergewaltigung setzen ein $\left(R_{\mathrm{kog}}\right)$. Der Patient fühlt sich bedroht, erlebt Panik und starken Ekel $\left(\mathrm{R}_{\mathrm{emo}}\right)$. 
Tab. 1. Häufigkeit und Schweregrad der Symptome (Mittelwerte, Range: 0-4) gemessen mit der PTSD-Symptom-Skala [Steil und Ehlers, 1992] im Therapieverlauf

\begin{tabular}{|c|c|c|c|c|c|c|c|}
\hline \multirow[t]{2}{*}{ Messzeitpunkte } & \multicolumn{2}{|c|}{ Intrusionen } & \multicolumn{2}{|c|}{ Vermeidung } & \multicolumn{2}{|c|}{ Hyperarousal } & \multirow[t]{2}{*}{ PTSD-Diagnose $^{\mathrm{a}}$} \\
\hline & $\mathrm{H}$ & $S$ & $\mathrm{H}$ & S & $\mathrm{H}$ & $\mathrm{S}$ & \\
\hline Prämessung & 3,80 & 3,60 & 2,86 & 3,29 & 3,40 & 3,80 & $\mathrm{Ja}$ \\
\hline Verlaufsmessung ${ }^{\mathrm{b}}$ & 2,60 & 3,00 & 2,71 & 2,71 & 3,20 & 3,40 & $\mathrm{Ja}$ \\
\hline Postexpositionsphase $^{c}$ & 1,80 & 1,60 & 1,29 & 1,29 & 2,40 & 1,60 & $\mathrm{Ja}$ \\
\hline Follow-up (18 Monate) & 0,80 & 0,80 & 0,14 & 0,29 & 0,60 & 0,80 & Nein \\
\hline
\end{tabular}

$\mathrm{H}=$ Häufigkeit; $\mathrm{S}$ = Schweregrad.

${ }^{a}$ Nach DSM-IV [American Psychiatric Association, 1996].

${ }^{\mathrm{b}}$ Zwischen Sitzung 48 und 49 (Messung erhoben für vom Bundesamt für Migration angeforderten Arztbericht).

${ }^{\mathrm{C}}$ Nach Sitzung 69.
Er dissoziiert $\left(\mathrm{R}_{\mathrm{emo}}\right)$ und liegt eine Weile erstarrt auf dem Bett $\left(\mathrm{R}_{\mathrm{mot}}\right)$. Anschließend duscht er lange kalt $\left(\mathrm{R}_{\mathrm{mot}}\right)$. Dies hilft, die Dissoziation zu beenden sowie die Intrusionen und Ekelgefühle zu reduzieren $\left(\mathrm{K}_{\text {kurz }}\right)$. Das Gefühl relativer Sicherheit tritt ein $\left(\mathrm{K}^{+}{ }_{\text {kurz }}\right)$. Die Intrusionen und Dissoziationen verunsichern den Patienten längerfristig; er befürchtet, verrückt zu werden $\left(\mathrm{K}_{\text {lang }}\right)$. Er sieht keinen Sinn mehr in seinem Leben und wünscht sich, im Krieg gestorben zu sein $\left(\mathrm{K}_{\text {lang }}^{-}\right)$. Durch das häufige Duschen hat er Ekzeme am Körper $\left(\mathrm{K}_{\text {lang }}\right)$. Seine Mitbewohner und die Betreuer des Durchgangszentrums ärgern sich zudem über ihn, weil er so häufig das Bad blockiert $\left(\mathrm{K}_{\text {lang }}\right.$ ). Langfristig werden durch die starken Intrusionen die erlernte Hilflosigkeit und die traumabezogenen dysfunktionalen Grundannahmen gestärkt, was die Symptomatik aufrechterhält ( $\left.\mathrm{K}_{\text {lang }}^{-}\right)$.

\section{Therapieplan}

Zur schnellstmöglichen effektiven Behandlung der PTSD-Symptomatik sollen kognitiv-verhaltenstherapeutische Verfahren eingesetzt werden. Zuerst soll dem Patienten in einer ausführlichen Psychoedukation die Entstehung und Aufrechterhaltung der Störung und assoziierter Probleme verdeutlicht werden. Anschließend sollen die subjektiv wichtigsten, intrusiv wiedererlebten Traumata mit verlängerter Exposition in sensu [Foa und Rothbaum, 1998] behandelt werden. Zur Reduktion des alltäglichen Vermeidungsverhaltens und der Entkräftung der Trigger soll der Patient zusätzlich zu Expositionen in vivo angeleitet werden [Foa und Rothbaum, 1998]. Die Behandlung soll nötigenfalls durch das Einüben antidissoziativer Strategien sowie den antidepressiven Aktivitätsaufbau unterstützt werden. Falls sich die assoziierte zwanghafte Symptomatik nicht durch die erfolgreiche PTSD-Behandlung reduziert, sollen zwangsspezifische Konfrontationen mit Reaktionsverhinderung durchgeführt werden.

Da der Patient als Asylbewerber einen unsicheren Aufenthaltsstatus hat und fremd in der Schweiz ist, muss von verschiedenen Zusatzstressoren (drohende Abschiebung, Arbeitslosigkeit, schwierige Wohnverhältnisse) während der Therapie ausgegangen werden. Solche Faktoren können die traumafokussierte Therapie behindern und Stabilisierungsmaßnahmen nötig machen [NICE, 2005]. Dennoch soll frühzeitig traumafokussiert - und nur wenn nötig stabilisierend - gearbeitet werden.

\section{Therapieverlauf}

$\mathrm{Zu}$ Therapiebeginn (Sitzungen 1-13) waren aufgrund der starken Anspannung und des ausgeprägten Misstrauens Sitzungen von nur ca. 30 min möglich. Der Patient vermied jeglichen Blickkontakt, war sichtlich übererregt und schwitzte stark. Er weigerte sich, über seine traumatischen Erfahrungen zu sprechen. Um dem Patienten Kontroll- und Sicherheitsgefühle zu ermöglichen und eine tragfähige Therapiebeziehung aufzubauen, konnte er in dieser Therapiephase den Großteil der Sitzungsinhalte selbst bestimmen. Gleichzeitig wurden ressourcenaktivierende Maßnahmen eingesetzt, wie der Aufbau von Tagesstruktur und von Aktivitäten (insbesondere die Wiederaufnahme von Sport und eines Deutschkurses) sowie das Einüben antidissoziativer Techniken. Parallel wurde psychoedukativ mit dem Patienten ein Störungsmodell (Teufelskreis der Angst [Müller, 2007]) und ein Veränderungsmodell (Schrankbeispiel [Ehlers, 2000, p. 33]) entwickelt, die die Notwendigkeit traumafokussierten Vorgehens - trotz erschwerter Bedingungen durch seine aktuell unsichere Lebenssituation - verdeutlichten. In dieser Zeit versuchte die Therapeutin erfolglos, bei den Behörden eine verbesserte Wohnsituation des Patienten zu erreichen, da die Heimunterbringung die Traumabearbeitung erschwerte (siehe Verhaltensanalyse). Dennoch wurde ab Sitzung 14 begonnen, mittels verlängerter Exposition [Foa und Rothbaum, 1998] traumafokussiert zu arbeiten. Vor der 16. Sitzung kam es aber infolge einer Polizeirazzia im Durchgangszentrum zu einer massiven Symptomsteigerung des Patienten. Statt traumafokussiert konnte deswegen in den Sitzungen 16 und 17 lediglich ressourcenaktivierend und antidissoziativ gearbeitet werden. Vor der 18. Sitzung fand der Patient eine befristete Anstellung als Fachkraft in der Gastronomie - eine solche Anstellung hat für Asylbewerber in der Schweiz aufenthaltsrechtliche Konsequenzen. Trotz Zustandsstabilisierung weigerte sich der Patient in den nächsten Sitzungen, traumafokussiert weiterzuarbeiten. Er befürchtete, die $\mathrm{Zu}$ satzbelastung durch die Traumaarbeit könne sich negativ auf seine Arbeit auswirken. Mit dem Patienten wurde die Zweischneidigkeit einer Unterbrechung besprochen: Der Aufrechterhaltung der PTSD-Symptomatik steht die Nützlichkeit des Pausierens bei unvorhersehbar verschobenen Prioritäten in seinem Leben gegenüber. Es wurde vereinbart, die Expositionstherapie weiterhin auszusetzen, aber baldmöglichst wieder aufzunehmen. Situationsbezogen wurde bis zur 20. Sitzung am Aufbau sozialer Kompetenzen (Gespräche führen, Blickkontakt halten) und an der kognitiven Neubewertung traumabezogener dysfunktionaler Kognitionen («Ich bin ein beschädigter Mensch», «Jeder sieht mir an, was mir passiert ist») gearbeitet. Der Patient fand eine eigene Wohnung und erlebte erstmals seit Jahren eine stabile, symptomreduzierte Phase. Diese Phase endete abrupt vor der 21. Sitzung. Erstens wurde der Arbeitsvertrag des Patienten nicht verlängert; zweitens erhielt er einen behördlichen Bescheid, dass sein Aufenthaltsstatus erneut überprüft werden müsse. Infolge dieser Ereignisse wurde der Patient suizidal und musste engmaschig betreut werden (Sitzungen 21-24). Sein Zustand verbesserte sich vor der 25. Sitzung, da der Patient zu diesem Zeitpunkt eine um 12 Monate verlängerte Aufenthaltsbewilligung erhielt und zudem eine unbefristete Anstellung als Hilfskraft in der Gastronomie fand. Ab der 26. Stunde konnte deswegen weiter traumafokussiert gearbeitet werden.

Während der kognitiven Intervention (Sitzungen 18-20) war die hohe Relevanz des gesamten biografischen Kontexts für die Aufrechterhaltung der Symptome deutlich geworden. Zudem hatte der Patient die in Sitzungen 14-15 durchgeführten verlängerten Expositionen aufgrund starker Belastung abbrechen wollen. Deswegen wurde ein Strategiewechsel der Traumatherapie vereinbart. Statt hierarchisch sollten die Traumata konsekutiv mithilfe Narrativer Expositionstherapie (NET) [Schauer et 
al., 2005] bearbeitet werden. Besondere Bedeutung innerhalb der NET (Sitzungen 26-44) kam den Konfrontationen mit dem Tod des ältesten Bruders und mit der Vergewaltigung zu. Da die Vergewaltigung nicht nur angst-, sondern auch schamhaft verarbeitet war, wurden die Expositionen in sensu durch kognitive Methoden und Expositionen in vivo (unter anderem Spiegelkonfrontation, sich selbst berühren [vgl. Boos, 2005]) unterstützt (Sitzungen 45-48). Bei deutlichem Symptomrückgang wünschte sich der Patient, ab Sitzung 49 das Setting aufzulockern. Alltagsrelevante Themen (wie der Umgang mit anderen Menschen, Partnerschaft, Sexualität, Weiterbildung) standen im Vordergrund dieser Therapiephase. Nur wenn sich einzelne Intrusionen verstärkten, wünschte sich der Patient gelegentlich wieder Expositionen in sensu und in vivo. Diese stabile Phase endete erneut abrupt: Eine schwere Erkrankung des in Bosnien lebenden Bruders und eine erneute Überprüfung seines Aufenthaltsrechts lösten eine Symptomexazerbation beim Patienten aus. Der Patient überwand die Krise mithilfe engmaschiger Betreuung (Sitzungen 62-68) schnell. Er entschloss sich zudem, heimlich nach Bosnien einzureisen. In Bosnien waren sein Bruder und dessen Frau dankbar für seine Anwesenheit. Zudem konnte der Patient sich seinen Wunsch erfüllen, Grabsteine auf den Gräbern der älteren Brüder aufzustellen. Er kehrte mit dem Gefühl, gebraucht zu werden, und dem Wissen, seine Pflicht getan zu haben, in die Schweiz zurück.

Zur Nachsorge fanden ab der 69. Sitzung über 1 Jahr hinweg bedarfsweise 7 maximal halbstündige Treffen statt. In diesen Treffen schilderte der Patient seine aktuelle Lebenssituation; wenn nötig wurden funktionale Strategien zum Umgang mit schwierigen Situationen erarbeitet (z.B. der Freundin von der Vergewaltigung erzählen), die der Patient selbständig umsetzte.

\section{Aktuelle Situation}

Der Patient hat eine feste Anstellung im Gastronomiegewerbe und lebt in einer eigenen Wohnung. Er hat eine Freundin (seit Sitzung 49); das Paar plant zu heiraten. Der Patient hat sich zudem einen Bekanntenkreis aufgebaut, mit dem er in seiner Freizeit Unternehmungen macht. Er spricht mittlerweile gut Deutsch, nimmt jedoch weiterhin regelmäßig an Deutschkursen teil. Er macht eine Ausbildung zur Fachkraft in der Gastronomie und besucht parallel verschiedene Kurse (z.B. Feuerwehr, Führerschein). Im April 2008 erhielt er eine sichere, wenngleich befristete Aufenthaltserlaubnis. Im Juni 2008 machte der Patient Ferien in Bosnien. Zur Therapeutin erhält er einen unregelmäßigen telefonischen Kontakt aufrecht.

\section{Diskussion}

Die im Therapieplan formulierten Ziele wurden im Lauf dieser ambulanten, 3 Jahre dauernden Therapie erreicht. Beim Follow-up nach 1,5 Jahren erfüllte der Patient die PTSD-Kriterien nicht mehr. Er berichtete, nur noch selten Traumaerinnerungen und Alpträume zu haben, die im Vergleich zu früher «viel ferner» und wenig belastend seien. Dissoziative, depressive und zwanghafte Symptome wurden vom Patienten nicht mehr erlebt. Dieser Symptomrückgang ermöglichte dem Patienten im Therapieverlauf, sein Leben sukzessiv neu zu gestalten. Zusätzlich zum Aufbau persönlicher Beziehungen gelang es ihm, Integrationsleistungen zu vollbringen, die vor der Therapie undenkbar gewesen wären. Aufgrund PTSDsymptomatisch ausgeprägten Misstrauens und negativer Therapievorerfahrungen war der Aufbau der therapeutischen Beziehung anfangs sehr schwierig. Insbesondere war für den Patienten die Vorstellung, seine traumatischen Erfahrungen detailliert schildern zu müssen, extrem belastend. Im Therapieverlauf konnte der Patient jedoch Vertrauen fassen, so dass eine konstruktive Arbeitsatmosphäre entstand.

Die Behandlung traumatisierter Flüchtlinge und Asylbewerber gilt aus verschiedenen Gründen als schwierig. Sicherlich mag bei vielen traumatisierten Flüchtlingen und Folteropfern die von Basoglu [2006] geforderte traumafokussierte Kurzzeittherapie erfolgreich und ausreichend sein. Gleichzeitig wird es aber auch viele Patienten geben, mit denen aufgrund ihrer schweren Symptomatik oder belastender Lebensumstände nicht von Anfang an konsequent traumafokussiert gearbeitet werden kann und die eine längerfristige Anbindung benötigen. Verschiedene Autoren betonen, dass dies jedoch nicht notwendigerweise eine Kontraindikation für eine störungsspezifische traumafokussierte Therapie ist [z.B. Basoglu, 2006; Grey und Young, 2007]. Auch sollten akute Krisen nicht zum Abbruch traumafokussierten Vorgehens führen, sondern im Sinne des empfohlenen zirkulären Phasenwechsels [NICE, 2005] lediglich eine Unterbrechung darstellen.

Trotz erwiesener Wirksamkeit haben es traumafokussierte Verfahren äußerst schwer, sich in der Praxis durchzusetzen [Becker et al., 2004]. Dies mag auch damit zusammenhängen, dass die meisten dieser Verfahren manualisiert vorliegen und viele Psychotherapeuten dem Gebrauch von Manualen skeptisch gegenüber stehen [Addis und Krasnow, 2000]. Erste Befunde zeigen jedoch, dass die flexible Anwendung kognitiv-verhaltenstherapeutischer PTSD-Therapien ebenso erfolgreich ist [Levitt et al., 2007; Schulz et al., 2006] und nicht notwendigerweise länger dauert [Schulz et al., 2006] als das manualgetreue Vorgehen.

Dieser Fallbericht schildert ein solches nicht manualgetreues Vorgehen mit mehrfachen Phasenwechseln. Dabei wurde versucht, eine Balance zwischen dem beharrlichen Festhalten an traumafokussiertem Vorgehen und dem Eingehen auf Bedürfnisse des Patienten herzustellen. Die Expositionen wurden nur unterbrochen, wenn der Patient die Weiterbehandlung verweigerte oder Extrembelastungen - wie die Prüfung des Aufenthaltsstatus durch die Behörden - vorlagen. Das Einverständnis, von einer Stabilisierungs- in eine Expositionsphase zurückzuwechseln, schien für den Patienten von folgenden Faktoren abhängig: ein nachvollziehbares Therapiemodell, die gemeinsame zeitliche Abstimmung des Phasenwechsels sowie eine gute Therapiebeziehung.

Trotz des Therapieerfolgs ist selbstverständlich unklar, ob teilweise das Festhalten an begonnenen Phasen und Verfahren indiziert gewesen wäre oder ob - im Gegenteil - der frühere Wechsel zu einer anderen Phase oder zu einem anderen Verfahren schneller zum Erfolg geführt hätte. Limitierend ist zudem hinzuzufügen, dass eine umfangreichere Diagnostik zur Abbildung der Veränderung der somatischen und depressiven Symptomatik zusätzlich zur PTSD-Kernsymptomatik wünschenswert gewesen wäre.

Zusätzlich zur erfolgreichen Symptomreduktion demonstriert der Fallbericht, dass es auch aufenthaltsrechtlich äu- 
ßerst sinnvoll sein kann, frühzeitig mit der Traumatherapie zu beginnen: Aufgrund des Symptomrückgangs konnte der Patient rascher Deutsch lernen und einer Arbeit nachgehen. So konnte er die behördlicherseits geforderten Integrationsbemühungen nachweisen und erhielt eine vorübergehende Aufnahme als Flüchtling.

Im Nachgespräch fasste der Patient die Konfrontationstherapie folgendermaßen zusammen: «Es ging mir jedes Mal 3 Tage lang schlecht nach der [Expositions-]Therapie, dann ging es mir 4 Tage besser, und dann musste ich wieder in die Therapie, und ich wusste, es wird wieder schlimm. [...] Ich habe Sie gehasst. Und ich dachte jedes Mal, das ist das letzte Mal, dass ich komme. Aber dann merkte ich, dass es das Einzige ist, was mir hilft, und ich bin wieder in die Therapie gegangen. [...] Und jetzt weiß ich, dass Sie mir das Leben gerettet haben, und ich bin endlich wieder ich selbst.»

\section{Schlussfolgerungen}

Trotz zunehmender Forschung zur Psychotherapie traumatisierter Kriegs- und Folteropfer ist die wissenschaftliche Datenlage nach wie vor erschreckend spärlich. Randomisierte kontrollierte Studien sind dringend nötig, welche die Effektivität therapeutischer Verfahren vergleichen, die bei Bedarf flexibel an die schwierigen Lebensbedingungen dieser Menschen angepasst werden können.
Wie der Fallbericht beispielhaft zeigt, können Patienten mit schwerer Symptomatik auch unter anhaltend schwierigen Lebenssituationen traumafokussiert behandelt werden. Da während der Therapie Krisen auftreten können, ist die konsequente Anwendung manualisierter Verfahren jedoch erschwert und hat auch nicht notwendigerweise höchste Priorität. Ein phasenorientiertes Vorgehen, das flexibel an die jeweilige aktuelle Lebenssituation jedes einzelnen Patienten angepasst wird, scheint beim heutigen Wissensstand eine sinnvolle und pragmatische Lösung. Trotz flexibler Anwendung sollte auch individuell angepasstes, phasenorientiertes Vorgehen gut durchdacht sein. Gleichzeitig sollte es zielgerichtet auf eine schnelle Traumaexposition hinzielen, die das entscheidende therapeutische Mittel der Traumatherapie ist. Wünschenswert wäre dazu eine schulenübergreifende, undogmatische Methodenvielfalt seitens der Therapeuten. So könnten nach ausreichender Diagnostik, angelehnt an die internationalen Leitlinien, evidenzbasierte traumafokussierte Therapieverfahren ausgewählt werden, die bei Bedarf flexibel durch Stabilisierungsmaßnahmen ergänzt werden.

\section{Dank}

Der Patient ist mit der Veröffentlichung seiner Behandlungsgeschichte einverstanden. Ich möchte mich hiermit bei ihm für seinen Mut, seine Ausdauer und sein Vertrauen bedanken.

\section{Literatur}

Addis ME, Krasnow AD: A national survey of practicing psychologists' attitudes toward psychotherapy treatment manuals. J Consult Clin Psychol 2000;68: 331-339.

Ai AL, Peterson C, Ubelhor D: War-related trauma and symptoms of posttraumatic stress disorder among adult Kosovar refugees. J Trauma Stress 2002;15: 157-160.

American Psychiatric Association: Diagnostisches und Statistisches Manual Psychischer Störungen. DSMIV. Göttingen, Hogrefe, 1996.

Australian Centre for Posttraumatic Mental Health: Australian guidelines for the treatment of adults with acute stress disorder and posttraumatic stress disorder. Melbourne, Australian Centre for Posttraumatic Mental Health, 2007.

Basoglu M: Rehabilitation of traumatised refugees and survivors of torture. BMJ 2006;333:1230-1231.

Becker CB, Zayfert C, Anderson E: A survey of psychologists' attitudes towards and utilization of exposure therapy for PTSD. Behav Res Ther 2004;42: 277-292.

Bichescu D, Neuner F, Schauer M, Elbert T: Narrative exposure therapy for political imprisonment-related chronic posttraumatic stress disorder and depression. Behav Res Ther 2007;45:2212-2220.

Bisson J, Andrew M: Psychological treatment of posttraumatic stress disorder (PTSD). Cochrane Database Syst Rev 2007;(3):CD003388.

Boos A: Kognitive Verhaltenstherapie chronischer Traumatisierung. Ein Therapiemanual. Göttingen, Hogrefe, 2005.
Bradley R, Greene J, Russ E, Dutra L, Westen D: A multidimensional meta-analysis of psychotherapy for PTSD. Am J Psychiatry 2005;162:214-227.

Davidson JR, Hughes D, Blazer DG, George LK: Posttraumatic stress disorder in the community: an epidemiological study. Psychol Med 1991;21:713-721.

de Jong JT, Komproe IH, Van Ommeren M, Es Masri M, Araya M, Khaled N, van de Put W, Somasundaram D: Lifetime events and posttraumatic stress disorder in 4 postconflict settings. JAMA 2001;286: 555-562.

Ehlers A: Posttraumatische Belastungsstörungen. Göttingen, Hogrefe, 2000.

Fazel M, Wheeler J, Danesh J: Prevalence of serious mental disorder in 7000 refugees resettled in western countries: a systematic review. Lancet 2005;365: 1309-1314.

Fischer G, Becker-Fischer M: Stabilisierung vor Konfrontation in der Traumatherapie - Grundregel oder Mythos? Kommentar zum Beitrag von Frank Neuner (2008), in Verhaltenstherapie, 18(2), 109-118. Z Psychotraumatol Psychotherapiewiss Psychol Med 2008;6:85-94.

Fischer G, Reddemann L, Barwinski-Fäh R, Bering R: Traumaadaptierte tiefenpsychologisch fundierte und analytische Psychotherapie. Definition und Leitlinien. Psychotherapeut 2003;48:199-209.

Foa EB, Rothbaum BO: Treating the Trauma of Rape. New York, Guilford Publications, 1998.

Foa EB, Keane TM, Friedman MJ: Effective Treatments for PTSD: Practice Guidelines from the International Society for Traumatic Stress Studies. New York, Guilford, 2004
Foa EB, Riggs DS, Dancu CV, Rothbaum BO: Reliability and validity of a brief instrument for assessing post-traumatic stress disorder. J Trauma Stress 1993;6:459-473.

Gavagan T, Brodyaga L: Medical care for immigrants and refugees. Am Fam Physician 1998;57:10611068.

Grey N, Young K: Cognitive behaviour therapy with refugees and asylum seekers experiencing traumatic stress symptoms. Behav Cogn Psychother 2007;36: 3-19.

Hinton DE, Chhean D, Pich V, Safren SA, Hofmann SG, Pollack MH: A randomized controlled trial of cognitive-behavior therapy for Cambodian refugees with treatment-resistant PTSD and panic attacks: a cross-over design. J Trauma Stress 2005;18:617-629.

Hollifield M, Warner TD, Lian N, Krakow B, Jenkins JH, Kesler J, Stevenson J, Westermeyer J: Measuring trauma and health status in refugees: a critical review. JAMA 2002;288:611-621.

Levitt JT, Malta LS, Martin A, Davis L, Cloitre M: The flexible application of a manualized treatment for PTSD symptoms and functional impairment related to the 9/11 World Trade Center attack. Behay Res Ther 2007;45:1419-1433.

Miller GA, Elbert T, Rockstroh B: Judging psychiatric disorders in refugees. Lancet 2005;366:1604-1605; author reply 1605 .

Mollica RF, McInnes K, Sarajlić N, Lavelle J, Sarajlić I, Massagli MP: Disability associated with psychiatric comorbidity and health status in Bosnian refugees living in Croatia. JAMA 1999;282:433-439. 
Müller J: Kognitive Verhaltenstherapie mit Folterund Kriegsopfern; in Maier T, Schnyder U (Hrsg) Psychotherapie mit Folter- und Kriegsopfern. Bern, Huber, 2007.

National Institute for Health and Clinical Excellence (NICE): Post-traumatic stress disorder. The management of PTSD in adults and children in primary and secondary care. London, Royal College of Psychiatrists, 2005

Neuner F: Stabilisierung vor Konfrontation in der Traumatherapie - Grundregel oder Mythos? Verhaltenstherapie 2008;18:109-118.

Neuner F, Onyut PL, Ertl V, Odenwald M, Schauer E, Elbert T: Treatment of posttraumatic stress disorder by trained lay counselors in an African refugee settlement: a randomized controlled trial. J Consult Clin Psychol 2008;76:686-694.

Neuner F, Schauer M, Klaschik C, Karunakara U, Elbert T: A comparison of narrative exposure therapy, supportive counseling, and psychoeducation for treating posttraumatic stress disorder in an african refugee settlement. J Consult Clin Psychol 2004;72: 579-587.

Otto MW, Hinton D, Korbly NB, Chea A, Ba P, Gershuny BS, Pollack MH: Treatment of pharmacotherapy-refractory posttraumatic stress disorder among Cambodian refugees: a pilot study of combination treatment with cognitive-behavior therapy vs sertraline alone. Behav Res Ther 2003;41:1271-1276.

Paunovic N, Ost LG: Cognitive-behavior therapy vs exposure therapy in the treatment of PTSD in refugees. Behav Res Ther 2001;39:1183-1197.

Perkonigg A, Kessler RC, Storz S, Wittchen HU: Traumatic events and post-traumatic stress disorder in the community: prevalence, risk factors and comorbidity. Acta Psychiatr Scand 2000;101:46-59.

Schauer M, Elbert T, Gotthardt S, Rockstroh B Odenwald M, Neuner F: Wiedererfahrung durch Psychotherapie modifiziert Geist und Gehirn. Verhaltenstherapie 2006;16:96-103.

Schauer M, Neuner F, Elbert T: Narrative Exposure Therapy (NET). Göttingen, Hogrefe und Huber, 2005.

Schulz PM, Resick PA, Huber LC, Griffin MG: The effectiveness of cognitive processing therapy for PTSD with refugees in a community setting. Cogn Behav Pract 2006;13:322-331.

Steil R, Ehlers A: Die modifizierte PTSD-Symptom Scale (PSS-SR). Jena, Universität Jena, 1992.

Thulesius H, Hakansson A: Screening for posttraumatic stress disorder symptoms among Bosnian refugees. J Trauma Stress 1999;12:167-174.

Turner SW, Bowie C, Dunn G, Shapo L, Yule W: Mental health of Kosovan Albanian refugees in the UK. Br J Psychiatry 2003;182:444-448.

United Nations High Commissioner for Refugees: UNHCR Statistical Yearbook 2006. Trends in Displacement, Protection and Solutions. 15.01.2008. www.unhcr.org/statistics/STATISTICS/478cda572. html.

von Lersner U, Rieder H, Elbert T: Psychische Gesundheit und Rückkehrvorstellungen am Beispiel von Flüchtlingen aus dem ehemaligen Jugoslawien. Z Klin Psychol Psychother 2008;37:112-121.

Weine SM, Kulenovic AD, Pavkovic I, Gibbons R Testimony psychotherapy in Bosnian refugees: A pilot study. Am J Psychiatry 1998;155:1720-1726. 